\title{
Treatment of strabismus in adults with botulinum toxin $\mathrm{A}$
}

\author{
J S Elston, J P LEE, C M POWELl, C HOGG, AND P CLARK
}

From the Institute of Ophthalmology and Moorfields Eye Hospital, London

SUMMARY Eighty-five adults with horizontal concomitant strabismus were treated with an injection of a low dose of botulinum toxin A (BTXA) into the lateral or medial rectus muscle. The ocular deviation was reduced by an average of $60 \%$ independently of its size and whether or not surgery had previously been performed. The change was temporary, however, except in those cases with binocular functions, when fusion was re-established. Repeated low dose injections can maintain the improvement, but at higher doses, although larger reductions are produced, temporary local side effects-ptosis and vertical strabismus-are common. The technique is simple, well tolerated, and has no systemic side effects.

Most adults considered for strabismus surgery have long-standing unsightly ocular deviations associated with amblyopia, no demonstrable binocular functions, and a history of one or more eye muscle operations in childhood. ${ }^{1}$ The short term effect of further surgical treatment is unpredictable, and may reduce eye movements, while in the long term the strabismus frequently recurs. ${ }^{2}$ The concept of chemically modifying extraocular muscle function in such circumstances is attractive, and botulinum toxin A has the necessary chemical and biological properties. Its manufacture and quantitation have been standardised, and animal experiments have established that an appropriate dose injected directly into a skeletal muscle causes local paralysis without systemic toxicity. ${ }^{34}$ Individual extraocular muscles can be selectively weakened in the rhesus monkey, producing a strabismus, and although the treated muscles recover full function there is a permanent change in the ocular alignment. ${ }^{5}$ The toxin is immunologically inert at the doses used and can be repeatedly injected. ${ }^{6}$

The clinical application of botulinum toxin $\mathrm{A}$ was established in 1980 by Scott, ${ }^{7}$ who showed that an extraocular muscle in a patient with strabismus can be effectively weakened under local anaesthesia, allowing the deviated eye to move into alignment. Several hundred patients ranging in age from 5 months ${ }^{8}$ to 80 years have since been treated in the

Correspondence to Mr J S Elston, FRCS, Moorfields Eye Hospital, City Road, London EC1V 2PD.
United States under Scott's sponsorship, and no adverse systemic effects have been reported. The long term results, however, are not yet established. The results of the treatment of 85 unselected cases of adult strabismus with botulinum toxin $\mathrm{A}$ as an alternative to surgical treatment are reported. The follow-up period is from 12 to 20 months.

\section{Patients, materials, and methods}

Eighty-five patients aged 18 to 72 scheduled for surgery for horizontal concomitant strabismus accepted the alternative of treatment with botulinum toxin injections (Table 1). Informed consent was obtained, and the patients reserved the right to withdraw from the trial or ask for surgical treatment

Table 1 Clinical features of patients treated

\begin{tabular}{ll}
\hline Total & 85 \\
Male & 31 \\
Female & 54 \\
Age range & $18-72$ years \\
Average age & 40 years \\
Esotropia & 37 cases \\
Exotropia & 48 \\
Amblyopia & 46 \\
Previous surgery & 66 \\
Binocular functions demonstrable & 2 \\
Average pretreatment deviation & 50 prism dioptres \\
\hline
\end{tabular}

Amblyopia was defined as a difference between the two eyes of two or more lines Snellen chart best corrected acuity without structural abnormality of the fundus or anterior visual pathway. 
at any stage. There were 37 cases of esotropia and 48 cases of exotropia, 20 of whom had had surgery for esotropia. Sixty-six patients had undergone strabismus surgery in the past, 28 having a history of two or more operations to a maximum of five to correct their deviation (Table 1). The angle of strabismus was measured by prism cover test at near and distance fixation, or by corneal reflections if fixation was poor. Binocular functions were investigated and the range of eye movements noted.

Botulinum toxin $\mathrm{A}$ in ampoules containing $0.05 \mu \mathrm{g}$ freeze dried neurotoxin bound to haemagglutinin was reconstituted with $1.0 \mathrm{ml}$ preservative-free sterile saline, and then diluted to give either $3 \cdot 12 \times 10^{-4} \mu \mathrm{g}$ or $1.56 \times 10^{-3} \mu \mathrm{g}$ of neurotoxin in $0 \cdot 1$ $\mathrm{ml}$ of solution. Amethocaine 1\% local anaesthetic, 4 drops, was instilled in the conjunctival sac, and adrenaline $0 \cdot 25 \%, 1$ drop, to constrict the conjunctival vasculature and allow the extraocular muscle to be easily seen. The toxin was injected through a 27 gauge needle with polytetrafluoroethylene insulation except at the tip, which constituted the active electrode of a differential pair, the reference and ground electrodes being standard silver:silver chloride electrodes taped to the brow, with the reference electrode over the eye to be treated. The needle tip was passed through the conjunctiva, then posteriorly alongside the muscle until the equator of the globe was passed, when it was angled into the muscle. ${ }^{9}$ An electromyographic (EMG) signal was recorded from the needle tip and allowed the appropriate muscle to be identified when activated by the patient's voluntary gaze.

All patients were treated with a dose of $3.12 \times 10^{-4} \mu \mathrm{g}$ neurotoxin into the muscle (lateral or medial rectus) responsible for the deviation of the eye; orthoptic measurements were repeated at one, two, four, and eight weeks. If the initial response was inadequate, after its paretic effect had worn off, $1.56 \times 10^{-3} \mu \mathrm{g}$ neurotoxin was injected into the same muscle and the follow-up schedule repeated. When the appropriate dose of neurotoxin for the individual patient had been established, injections were repeated if the strabismus recurred to a cosmetically unsightly extent.

\section{STATISTICAL ANALYSIS}

Stepwise regression was used to determine whether any other variables could help to explain the initial angle of the strabismus or any changes in the angle. The initial angle and both the actual and proportional changes at one, two, four, and eight weeks were chosen as dependent variables. The age and sex of the patients, the direction of the strabismus (esotropia or exotropia), and previous surgery were used as explanatory variables. The initial size was also used as an explanatory variable when the changes in strabismus were used as dependent variables. A partial $F$ ratio of 4 was used to determine inclusion and exclusion of variables in the model.

\section{Results}

From March to December 1983, 85 patients were enrolled in the study. The ocular deviations were large, with a high incidence of amblyopia and attempted surgical correction, and binocular functions were demonstrable in only two (Table 1). The size of the initial strabismus was related to the age of patients, with older patient having larger deviations (Fig. 1). Stepwise regression showed that no other explanatory variable had any effect.

The ocular deviation was regarded as abolished if over the period of follow-up the cover test was

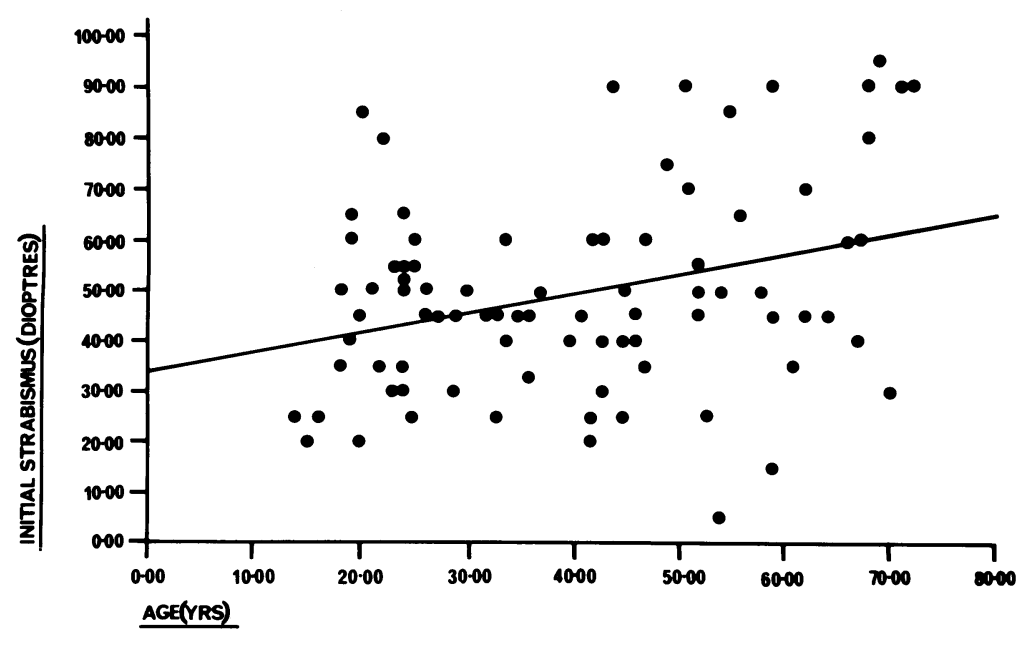

Fig. 1 Size of strabismus before treatment related to age of patient. 
Table 2 Mean reduction in strabismus over time in weeks

\begin{tabular}{|c|c|c|c|c|}
\hline & \multicolumn{4}{|l|}{ Weeks } \\
\hline & 1 & 2 & 4 & 8 \\
\hline Number of patients & 85 & 84 & 68 & 43 \\
\hline Actual (prism dioptres) & 23.46 & 34.04 & $29 \cdot 00$ & $24 \cdot 67$ \\
\hline SE & 1.87 & $2 \cdot 47$ & 2.76 & $2 \cdot 83$ \\
\hline Proportional & 0.43 & 0.62 & 0.61 & 0.46 \\
\hline$S E$ & 0.054 & 0.044 & 0.046 & $0 \cdot 163$ \\
\hline
\end{tabular}

$\mathrm{SE}=$ standard error of the mean.

negative and full binocular function demonstrable, and as acceptable if it was stable, measured no more than $50 \%$ of the pretreatment angle, and the patient was satisfied with the appearance. The deviation was designated recurrent if any measurement was greater than $50 \%$ of the pretreatment angle.

The maximum change in the angle of the strabismus after one injection occurred at two and four weeks, and averaged 34 prism dioptres (Table 2) but was larger in patients with larger pretreatment angles (Fig. 2). No change in the angle was recorded in two cases after treatment; the injection had not been made into the muscle, however, since no weakness was produced. The proportional change was relatively constant and averaged $60 \%$ of the original deviation (Fig. 3). The sex of the patients, the direction of the deviation, and previous surgery had no influence on the findings. At this stage the eye movements and saccadic velocity in the direction of action of the injected muscle were reduced. The proportional reduction in strabismus lessened over the next six weeks (Fig. 3), by which time full eye movements were restored and a further injection could be considered.

There were no systemic complications. In four cases local spread of paralytic effect within the orbit

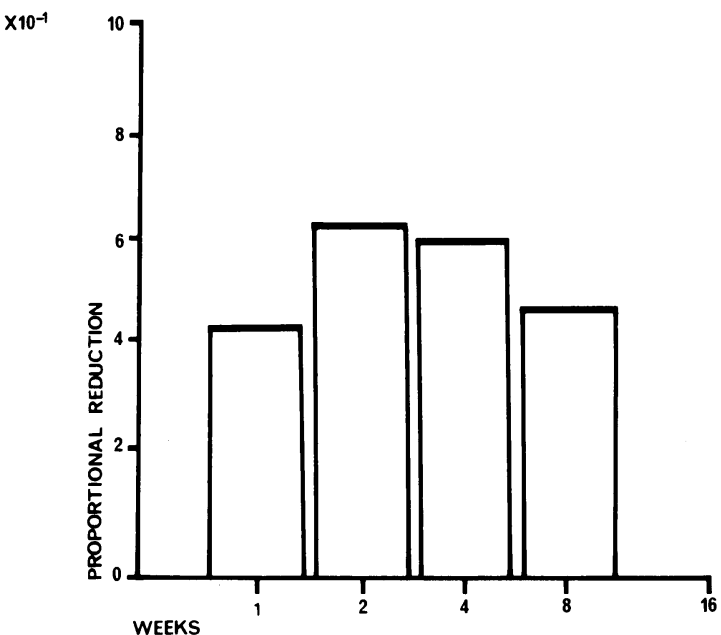

Fig. 3 Proportional reduction in strabismus declines from two weeks after the first injection.

was observed-an incomplete paralysis of the upper lid levator giving a partial ptosis in three cases, and an inferior rectus weakness in one-with complete recovery within six weeks. In 2 cases, with full binocular potential, the deviation was abolished by one injection.

\section{CASE I}

A 19-year-old girl suffered an attack of idiopathic inflammatory polyneuropathy (Guillain-Barré syndrome) in January 1983 associated with a right lateral rectus palsy. She recovered completely, but had a residual right esotropia of 60 prism dioptres associated with intermittent right suppression (Fig. 4A). The right medial rectus was treated with a single dose of $3.12 \times 10^{-4} \mu \mathrm{g} \mathrm{BTXA}$, producing a consecutive exotropia of 40 prism dioptres after two weeks, when
Fig. 2 Actual reduction in strabismus four weeks after first injection.

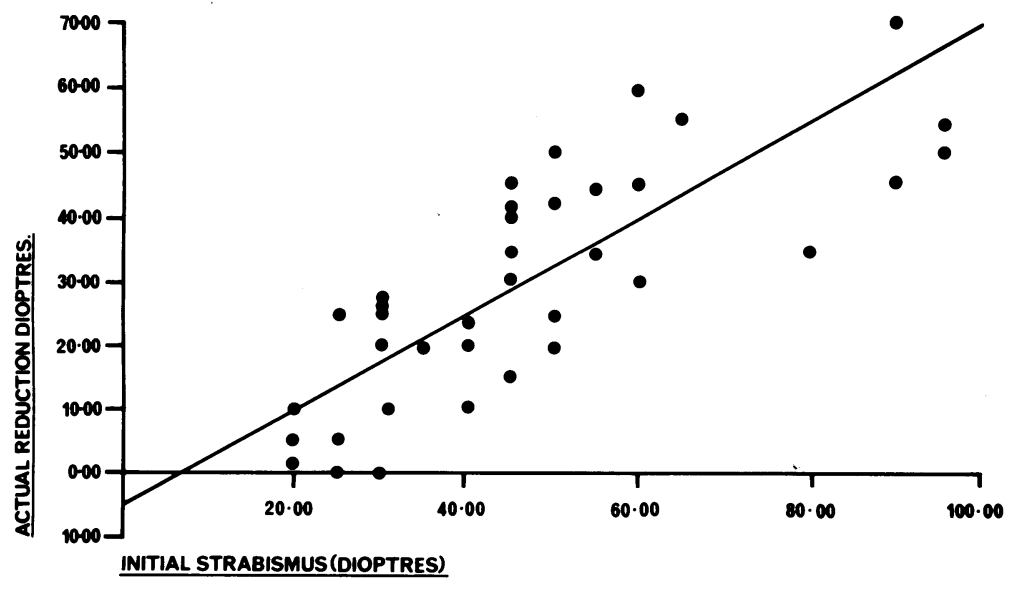


DIAGNOSIS:

No.
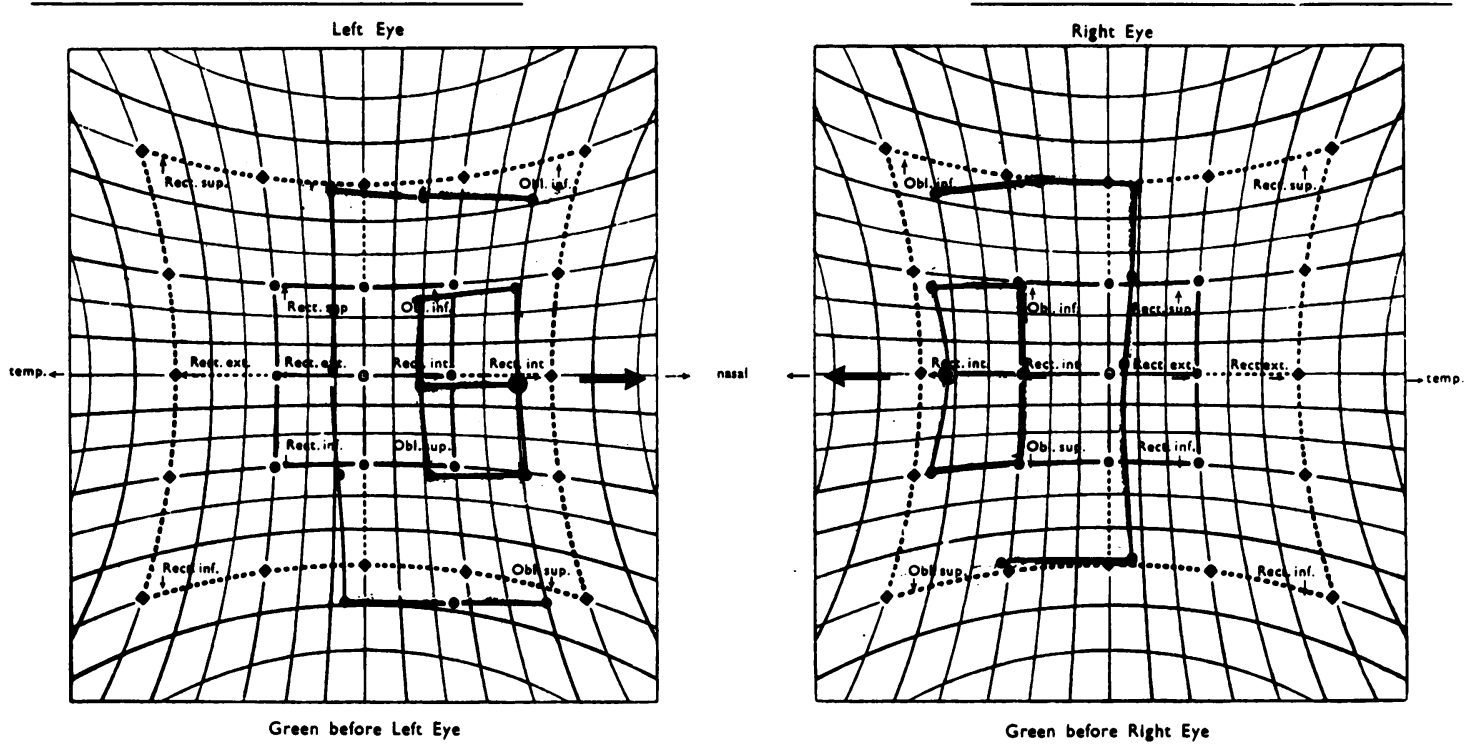

D 5

Fig. 4A

DIAGNOSIS:

No.
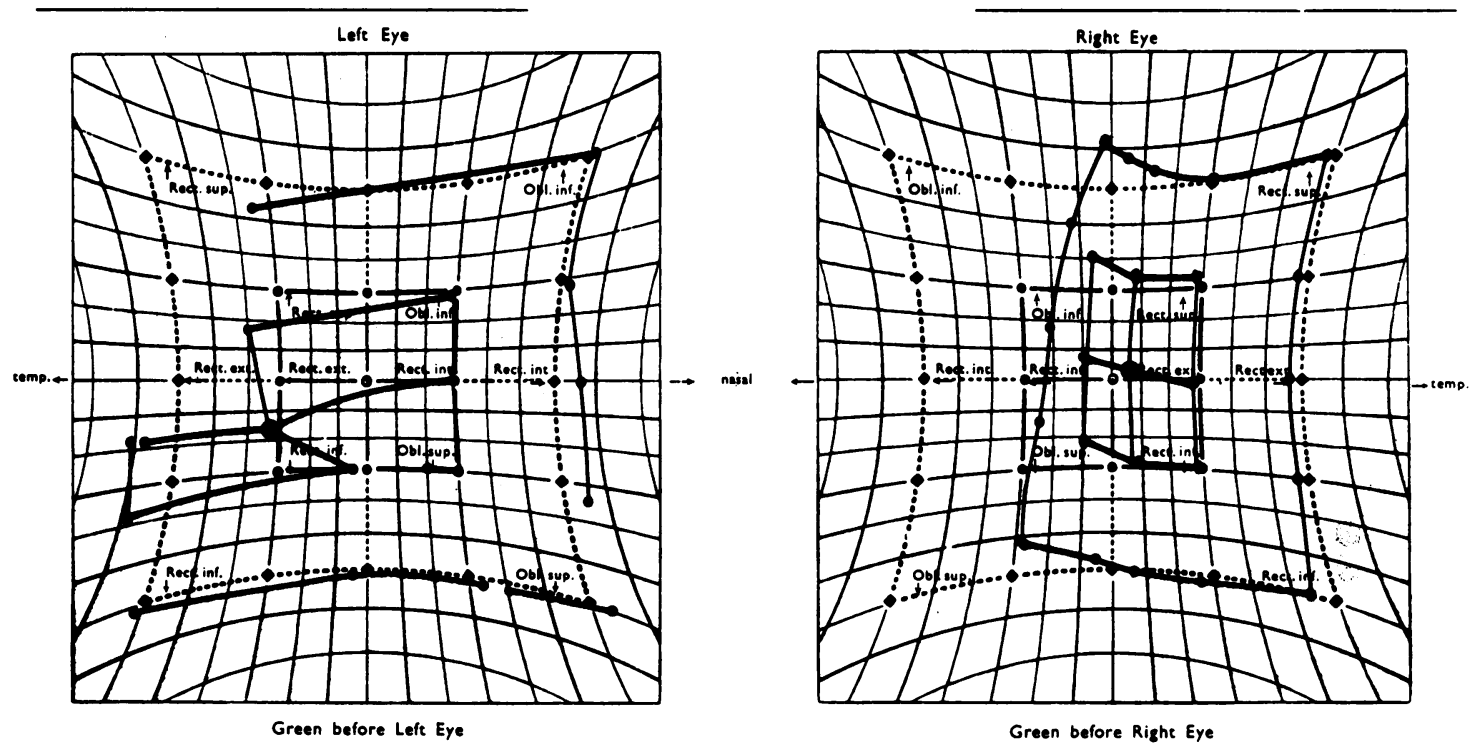

D 5

Fig. 4B

Fig. 4 Case 1. Serial Hess charts showing the re-establishment of normal binocular function. A: Pretreatment. B: Two weeks after treatment. C: Twelve weeks after treatment. 
No.

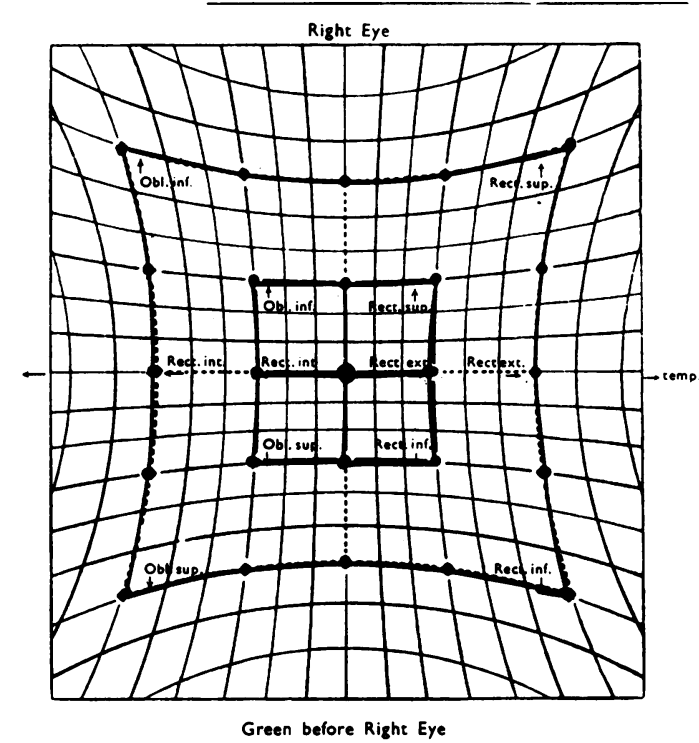

Green before Left Eye

D 5

Fig. 4C

binocular functions were re-established with the head turned to the left (Fig. 4B). Six weeks after the injection nearly full right adduction had returned, and at 12 weeks no abnormality of the oculomotor system was detectable (Fig. 4C).

Table 3 Treatment of recurrent deviations: high and low dose schedules

\begin{tabular}{|c|c|c|}
\hline & $\begin{array}{l}\text { High dose } \\
1.56 \times 10^{-3} \mu g \\
B T X A\end{array}$ & $\begin{array}{l}\text { Low dose } \\
3 \cdot 12 \times 10^{-4} \mu g \\
B T X A\end{array}$ \\
\hline Total number & 21 & 44 \\
\hline Average age (years) & $48 \cdot 5$ & $37 \cdot 5$ \\
\hline Average pretreatment & & \\
\hline deviation (prism dioptres) & 70 & 43 \\
\hline Esotropia & 6 & 24 \\
\hline Exotropia & 15 & 20 \\
\hline $\begin{array}{l}\text { Average maximum change } \\
\text { in deviation after second }\end{array}$ & 38.5 & 22.5 \\
\hline Side effects: partial ptosis & & \\
\hline or vertical deviation & 10 & 6 \\
\hline Deviation abolished & 2 & $\mathbf{0}$ \\
\hline $\begin{array}{l}\text { Deviation acceptable with } \\
\text { continued treatment }\end{array}$ & 9 & 36 \\
\hline $\begin{array}{l}\text { Average total injections } \\
\text { required per year for } \\
\text { maintenance }\end{array}$ & $2 \cdot 5$ & $3 \cdot 2$ \\
\hline $\begin{array}{l}\text { Withdrew from trial: } \\
\text { no further treatment } \\
\text { surgical treatment }\end{array}$ & $\begin{array}{r}10 \\
3\end{array}$ & $\begin{array}{l}4 \\
4\end{array}$ \\
\hline
\end{tabular}

In eight cases the deviation was reduced to an extent acceptable to the patient. Follow-up measurements have shown a gradual return to the pretreatment strabismus over three months. Eight of the remaining 75 patients declined any further treatment, while two accepted surgery and 65 further injections. Twenty-one patients were treated on the high dose schedule; they were older and had larger squints than those on the low dose schedule. The maximum change in the angle of strabismus achieved on the second injection into the previously treated muscle averaged 38.5 prism dioptres. There was a high incidence of temporary partial ptosis and vertical muscle underaction due to orbital spread of BTXA from the treated muscle (Table 3). Over half the patients declined further injections and withdrew from the trial or opted for surgical treatment. The strabismus was unexpectedly abolished, however, in two cases; in which binocular functions had not been demonstrated before treatment. Nine patients have maintained an acceptable reduction in the deviation, requiring an average of 2.5 injections over the first year of follow-up to do so.

On the low dose schedule there were fewer side effects, and only one-fifth of the patients either withdrew from the trial or asked for surgery. The second injection resulted in an average change of 22.5 prism dioptres. However, a larger number of injections has been required to achieve an acceptable reduction in strabismus. 


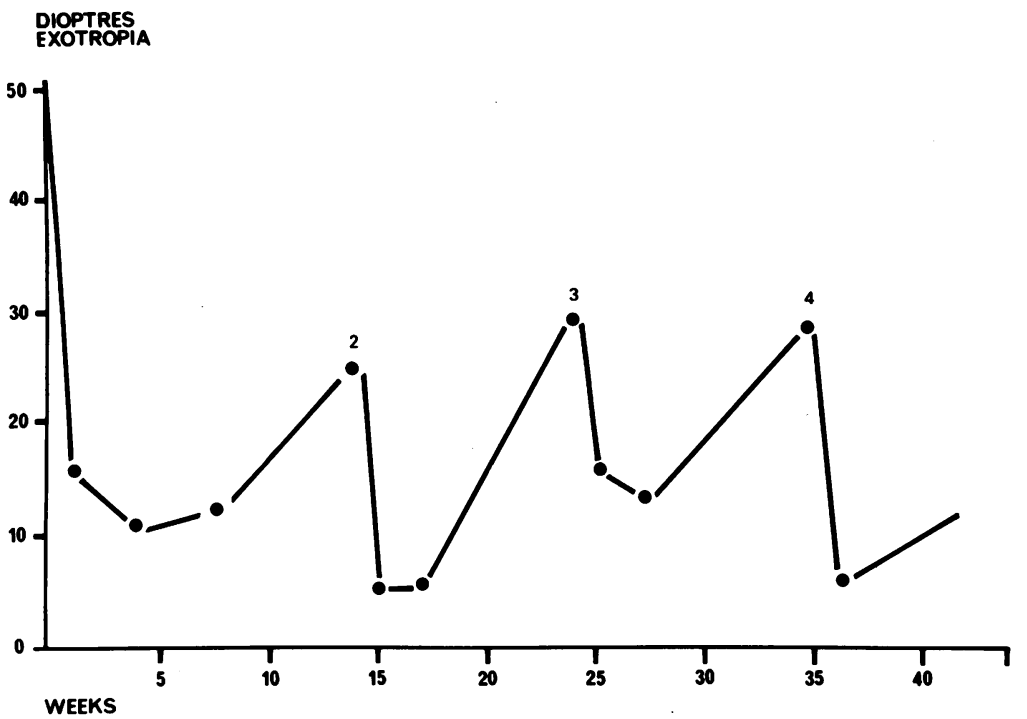

Fig. 5 Case 2. Treatment of recurrent consecutive exotropia by repeated injection (numbered) of $B T X A$ into the right lateral rectus.

CASE 2

An 18-year-old female student had treatment for a right convergent squint and amblyopia with glasses, patching, and three operations in childhood. At the age of 15 an operation for consecutive exotropia was carried out on the right eye, but the strabismus recurred and measured 50 prism dioptres. A total of four injections have been given into the right lateral rectus without side effects (Fig. 5).

\section{Discussion}

Motor nerve terminals contain acceptors which specifically bind botulinum toxin $\mathrm{A}$ and mediate its internalisation, where it prevents acetylcholine release, probably by lysosomal processing. ${ }^{10}$ The neuromuscular blockade is permanent, ${ }^{\text {" and muscle }}$ function recovers by the sprouting of new motor end plates from the axon, ${ }^{12}$ a process that takes longer in fast (twitch) skeletal muscles, which predominate in the extraocular muscles, than in slow (tonic) fibres. ${ }^{13}$ The intensity and duration of the flaccid paralysis of the extraocular muscle is therefore dependent on the dose of toxin injected. These results show that a single low dose of botulinum toxin A predictably alters the position of the eye within the orbit by selectively weakening the rectus muscle, independent of the size, direction, and previous treatment of the strabismus. If motor fusion is re-established, the deviation will be abolished. In general, however, the alteration is not sustained, and repeated injections are necessary. On the high dose schedule, in cases of large, longstanding strabismus, fibrosis and contracture of the muscle combined with reduced ocular movements and often exacerbated by previous surgery contribute to the poorly sustained response. The high incidence of side effects further limits the application of the technique in such cases. Repeated injections on the low dose schedule achieve a satisfactory stable improvement in younger patients with comparatively small deviations.

Two factors probably contribute to the sustained response: during the period of induced paralysis the ipsilateral antagonist rectus muscle develops contracture changes analogous to those sometimes seen in the medial rectus in cases of temporary lateral rectus palsy. ${ }^{14}$ At the same time, even after full recovery from the paralysis, there is a permanent disturbance of the pattern of motor innervation in the treated muscle. ${ }^{\text {is }}$ After repeated injections, the neuromuscular balance between the two opposing horizontal rectus muscles may be altered in favour of the untreated muscle and the strabismus reduced.

Surgical treatment of the patients in this study (average deviation 50 prism dioptres) would involve operating on two or three horizontal rectus muscles under general anaesthetic. Botulinum toxin treatment is shown here to be a safe and simple alternative, effective in younger patients with moderate to large strabismus. Longer follow-up will further define its therapeutic role.

The authors thank Mr Peter Fells for advice and encouragement, the staff of the Orthoptic Department for examining the patients, and Miss Karen Betts for typing the manuscript.

\section{References}

1 Duke-Elder S, Wybar KC. System of ophthalmology. London: Kimpton, 1973; 7: 487-512.

2 Dunlap EA. Overcorrections in horizontal strabismus surgery. In: Burian HM, Dunlap EA, Dyer JA, et al. Symposium on strabismus. St Louis: Mosby, 1971: 255-67. 
3 Guyton AC, MacDonald MA. Physiology of botulinum toxin. Arch Neurol 1947; 57: 578-92.

4 Ambache $\mathrm{N}$. The peripheral action of $\mathrm{Cl}$. botulinum toxin. J Physiol (Lond) 1949; 108: 127-41.

5 Scott AB, Rosenbaum AL, Collins CC. Pharmacological weakening of extraocular muscles. Invest Ophthalmol Vis Sci 1973; 2: 924-7.

6 Scott AB. Botulinum toxin injection into extraocular muscles as an alternative to strabismus surgery. Ophthalmology (Rochester) 1980; 87: 1044-9.

7 Scott AB. Botulinum toxin injection into extraocular muscles as an alternative to strabismus surgery. $J$ Pediatr Ophthalmol Strabismus 1980; 17: 21-5.

8 Magoon EH. Botulinum toxin chemo-denervation for strabismus in infants and children. $J$ Pediatr Ophthalmol Strabismus 1984; 21: 110-2.

9 Scott AB. Oculinum users manual. San Francisco: Smith Kettlewell Institute, 1984.
10 Dolly JO, Black J, Williams RS, Melling J. Acceptors for botulinum neurotoxin reside on motor nerve terminals and mediate its internalization. Nature 1984; 307: 457-60.

11 Tonge DA. Chronic effects of botulinum toxin on neuromuscular transmission and sensitivity to acetylcholine in slow and fast skeletal muscles of the mouse. J Physiol (Lond) 1974; 241: 127-39.

12 Duchen LW. An electron microscopic study of the changes induced by botulinum toxin in the motor end plates of slow and fast muscle fibres of the mouse. J Neurol Sci 1971; 144: 47-60).

13 Duchen LW. Changes in the electron microscopic structure of slow and fast skeletal muscle fibres of the mouse after local injection of botulinum toxin. J Neurol Sci 1971; 14: 60-74.

14 Duke-Elder S, Wybar KC. System of ophthalmology. London: Kimpton, 1973; 6: 650-7.

15 Duchen LW, Strich SJ. Demonstration of morphological abnormalities of motor nerve fibres after botulinum toxin injection in adult albino mice. J Physiol (Lond) 1967; 189: 2-4. 